\title{
A computational mechatronics approach for the analysis, synthesis and design of a simple active biped robot: Theory and experiments
}

doi:10.1533/abbi.2005.0053

\author{
L.-I. Lugo-Villeda ${ }^{1}$ and V. Parra-Vega ${ }^{2}$ \\ ${ }^{1}$ Mechatronics Research, Universidad Politécnica de Pachuca, Zempoala Hidalgo, México, CP 43830 \\ ${ }^{2}$ Mechatronics Division, Electrical Engineering Department, CINVESTAV-IPN, Av. IPN 2508, México DF, México
}

\begin{abstract}
Biped walking is a quite complex process that has been mastered only by human beings. Transferring this skill to a robot requires implementing advanced techniques in every aspect. To this end, a computational mechatronics platform was integrated to run the scheme for the analysis, synthesis and design to achieve planar biped walking. The result is an advanced computational tool that integrates advanced modeling and control as well as path planning techniques along with hardware-in-the-loop for perhaps the simplest biped robot. An experimental underactuated three-degree-of-freedom (two active and one passive) active biped robot yields encouraging results; that is, achieving biped walking with this simple device requires adding a telescopic support leg. Considering a more complete dynamic model to take into account frictional and contact forces.
\end{abstract}

Key words: Dynamic effects, computational mechatronics, biped walking, differential flatness, sliding modes.

\section{INTRODUCTION}

A simple model of a complex process is usually obtained by neglecting dynamic effects, which are considered unimportant for certain criteria under a range of operational conditions. Real-time control of such systems renders typically low performance under a wide range of conditions because the controller is designed for a locally valid model, and the realisation of such a model is therefore questionable: Can it be constructed? Does it have correlation to reality? Driven by this question, an experimental platform to run a computational mechatronics scheme for the analysis, synthesis and design of a simplified model of a complex task, such as the planar biped walking task, is integrated. This model has been considered previously by other authors, which is in fact an underactuated three degrees of freedom (two active and one passive) of an electromechanical system, considered to be the simplest active biped robot by recognised authors. However, it results in the inviability

Corresponding Author:

L.-I. Lugo-Villeda

Mechatronics Research

Universidad Politécnica de Pachuca

Pachuca-Cd. Sahagún Km 20, Zempoala Hidalgo

México, CP 43830

Email: lilugo@upp.edu.mx of building this biped, even when advanced controllers are designed, because the commercially available sensors and actuators cannot produce the very high acceleration needed to stabilise the biped, resulting in instability in the sagittal plane.

Dynamic 3D simulation and experimental data of the prototype are presented in this paper, which suggest that achieving biped walking with this simple device requires adding a telescopic support leg and considering a more complete dynamic model that also takes into account the frictional and contact forces. A fundamental tool for this analysis is the CAE simulator, integrated using several high-performance numerical and CAD packages, that implements dynamically a mechatronics approach to obtain the computational mechatronics scheme.

\section{Background}

Reproducing human-like biped walking in robots has attracted attention from a variety of research communities other than that of robotics itself, such as biomechanics, kinesiology, bionics and automatic control. Preliminary results indicate that a complex walking pattern arises for biped robots with a moving inertial frame. These biped robots are quite complex, and simplification of the electromechanical structure has been elusive. This problem poses the following question: Is there a simple biped robot architecture that yields stable walking patterns? Mother nature has mastered evolution to produce erect and stable 
biped walking in humans, in contrast to, for instance, quadruped walking in animals. Millions of years of evolution has shaped humans different from any living species, to give the leading edge through biped walking (Raibert 1986). This leads us to surmise that a simple biped robot may have difficulty achieving a stable walking pattern.

Recent studies on mechanically simple robots pose very interesting and challenging theoretical and technological problems, such as strange constraints (rolling wheel; Ioi $e t$ al. 2002), underactuated dynamics (Fantoni and Lozano 2002), chaotic behavior (González-Hernández 2004) and impact regime (Zheng and Hemami 1984). However, these systems usually require complex control systems, implemented of course under very fast sampling. What happens when the dynamic model does not reflect the real behavior? The system becomes instable. Thus, what is the limit of model simplification that reflects the complexity of a given robot task, such as biped walking? Can a very simple dynamic model of a biped robot be realised in real time? In this paper, we answer this particular problem using an advanced computational mechatronics scheme. To that end, we analize, synthesize and design a biped robot (Kieffer and Bale 1993) to find what the requirements are to walk in a real 3D world.

\section{A simple biped robot}

Biped walking is a very complex process, mastered by the human race through thousands of years of evolution. Therefore it is interesting to study biped walking and even more interesting to try to design the simplest robot that can achieve stable biped walking. We focus our attention on the simplest active biped model studied in the state-of-the-art literature, for example, the very simple planar three-degree-of-freedom (3-DOF) model proposed by Kieffer and Bale (1993), based on Raibert (1986). This model in theory walks in the vertical lane, neglecting any dynamics outside the $2 \mathrm{D}$ plane. The underlying assumptions of the dynamic model leads to three differential equations: two of them directly related to the two motors and the third to the torque difference between the two motors. The two legs, modeled as inverted pendulums, are connected by a central underactuated rotary body, which houses both motors. This is a very simple model of the very complex task of biped walking, in contrast to the very highly complex models of biped robots with torso, like the $S D R-4 X$ or $P 3$.

In particular, Spong et al. (2000) and McGee and Spong (2001) show that this simple biped robot is linearized under a change of coordinates, and a nonlinear control input can stabilise the system, whose zero dynamics governs the central body. Interestingly, Rouchon and Sira-Ramirez (2003) shows that flat and pole assignment stabilises the closedloop system; curiously, only kinematic simulations are presented. Furthermore, any implications in comparison to a physical system are not discussed. This is very interesting, prompting further studies to understand the realisation of such simple biped robots. Moreover, a careful analysis shows severe limitation, which may explain why nobody has ever attempted to build such an apparently simple system, even though very complex biped robots have been studied, for example, Grizzle (2001); Grizzle et al. (2000, 2001, 2002); Westervelt and Grizzle (2002); Plestan et al. (2001):

1. Constrained forces are ignored (implying that the biped robot is infinitesimally thin since point contact is assumed and that it is suspended on air, with no impact or other forces).

2. Dissipative frictional forces are neglected, as if no relative motion exists in contact parts.

3. At any given instant, one leg is in the air and the other on the ground. This produces a dynamic model coupled only through the rotary body, as if the swing leg were not attached to the stance leg. Besides that, there arises numerical problems because of commuting dynamics.

\section{Motivation}

Previous studies on biped walking show quite complex electromechanical systems; in contrast, recent studies have been on too simple biped models. However, while very complex biped robots have been built, such as the humanoid $P 3$ or the $S D R-4 X$, there are no real biped robots based on the simple model (Spong et al. 2000; McGee and Spong 2001; Rouchon and Sira-Ramirez 2003). In this realm, the discussion in the previous section leads us to consider an integral (mechatronics) approach to find out a realisable architecture of a simple biped mechanism.

\section{Contribution}

This article discusses the viability of building a simple model, and addresses the technological guidelines for building a feasible one. Dynamical 3D CAD-based simulations are provided, and an experimental prototype is built to support our conclusions. An advanced control system is introduced to yield robust and fast closed-loop response at a sampling period of $1 \mathrm{~ms}$. Strikingly, there is almost perfect matching between simulations and experiments, which was made possible by the integration of four commercial computer numerical packages into an advanced mechatronics computational platform. Five conclusions arise:

1. Since the model neglects any friction effects, there is no energy dissipation; thus this behaves in theory as a perpetual momentum machine, which is impossible to realise in practice.

2. The rotary body achieves very high acceleration in very small periods, requiring excellent electromechanical design and integration, which is very hard to achieve.

3. The model neglects the constrained forces that arise when switching from the swing leg to the stance leg. When the contact force vector is slightly off the normal, torsional torques induce instability and the biped falls down. 


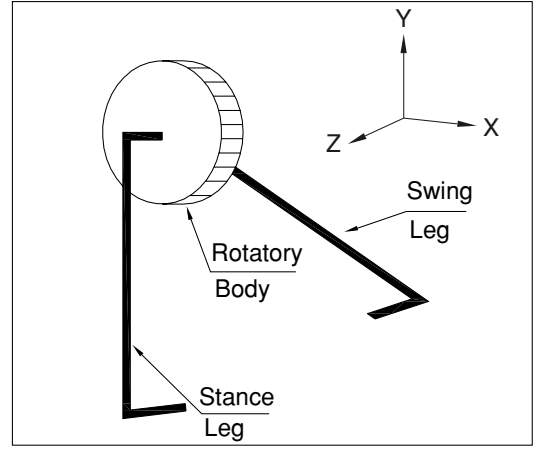

(a)

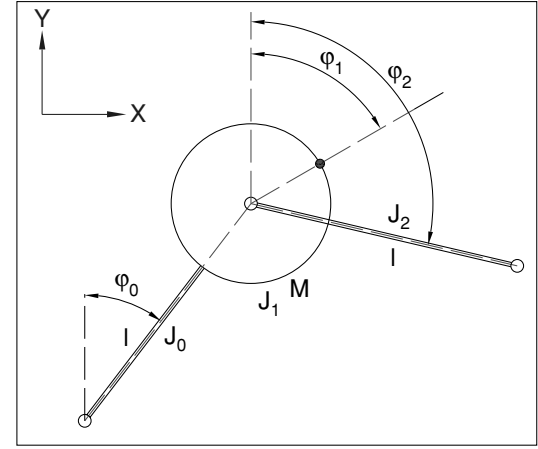

(b)

Figure 1 Three-degree-of-freedom biped robot: Perhaps the simplest mechanical system that might walk. (a) Biped robot architecture; (b) Absolute coordinates.

4. There is a surprising matching between the $3 \mathrm{D}$ CAD-CAE-based simulation and experimental results, suggesting that the computational mechatronics approach renders realistic data.

5. All these conclusions exhibit the high potential of $3 \mathrm{D}$ CAD-CAE-based simulations.

\section{Organization}

The next section presents the model and its kinematic constraints, and the control scheme is presented in the section 'Continuous Biped Walking'. The 3D CAD-CAEbased computational platform is presented in the section 'Mechatronics Computer Platform', and using it, the analysis of the biped is presented in the section 'The Real Biped Robot Prototype'. Experimental results are then discussed in the next section, followed by conclusions.

\section{DYNAMICS OF THE BIPED PLANAR ROBOT}

According to Kieffer and Bale (1993), a rotary central body interconnects, without any friction, two links or legs, stance leg and swing leg, in the sagittal plane (see Figure 1). Therefore, there are three DOFs, two of them of motion associated to the legs and the third to the rotary body. Modeling this system yields the following:

$$
\begin{aligned}
& \mathcal{F}_{0} \ddot{\varphi}_{0}=M g l \sin \varphi_{0} \tau_{1} \\
& \mathcal{F}_{1} \ddot{\varphi}_{1}=\tau_{1}-\tau_{2} \\
& \mathcal{F}_{2} \ddot{\varphi}_{2}=\tau_{2} .
\end{aligned}
$$

The variables are described in Table 1 . Note that the torque difference excites the rotary body at the inertial level; thus this arrangement may produce high acceleration. According to equations (1)-(3), some points are in order

1. This is a biped walking robot without knees (legs are two inverted pendulums of equal length).

2. The dynamics are constrained to the vertical plane (there are no forces along the $\vec{z}$ axis).

3. Point contacts are infinitesimally small, and somehow fixed (but no contact force is modelled, and no sliding or slipping is permitted).
Table 1 Description of the parameters

\begin{tabular}{lll}
\hline Symbol & Description & Units \\
\hline $\mathcal{F}_{0}$ & Stance leg discrete inertia & $\mathrm{kgm}^{2}$ \\
$\mathcal{F}_{1}$ & Hip discrete inertia & $\mathrm{kgm}^{2}$ \\
$\mathcal{F}_{2}$ & Swing leg discrete inertia & $\mathrm{kgm}^{2}$ \\
$\varphi_{0}$ & Stance leg angle & $\mathrm{rad}$ \\
$\varphi_{1}$ & Hip angle & $\mathrm{rad}$ \\
$\varphi_{2}$ & Swing leg angle & $\mathrm{rad}$ \\
$M$ & Pendulum mass & $\mathrm{kg}$ \\
$l$ & Leg length & $\mathrm{m}$ \\
$g$ & Gravity constant & $\mathrm{m} / \mathrm{s}^{2}$ \\
$\tau_{1}$ & Stance leg torque & $\mathrm{Nm}$ \\
$\tau_{2}$ & Swing leg torque & $\mathrm{Nm}$ \\
\hline
\end{tabular}

4. Intersection of the pendulums is fixed, but this point is moving in the plane, without a fixed inertial frame.

5. Coupling dynamics are neglected (Coriolis forces are not included).

6. For Step 1, one leg remains in contact while the other one balances in the air (for Step 2 and further, the model switches legs discontinuously).

7. Torques commute discontinuously (but the switching is not modeled).

8. Parameters also commute discontinuously.

9. The rotary body does not commute (but the input changes sign).

10. Contact and impact dynamics are not considered (perfect switching and perfect landing is assumed; perfect means the action is achieved without any collateral effect).

11. The rotary body is suspended somehow (there is no gravitational pull acting on it).

Finally, note that the motion of the biped robot is in the vertical plane, and there is no actuator to constrain it in the lateral plane. However, the real biped robot is necessarily composed of solid parts, with dimension in the $\vec{z}$ axis. Thus, if there is any momentum-force along the $\vec{z}$ axis, there may arise forces in the unitary axis $\vec{k}$, which may produce instability, causing the biped robot to fall. 
That is, the underlying assumption of this model is that the mechanical components are very thin and light, such that the cross term of the inertial tensor $I$ are neglected $\left(I_{x y}, I_{x z}, I_{y x}, I_{y z}, I_{z x}, I_{z y}\right.$, and $I_{z z}$ are zero).

\section{CONTINUOUS BIPED WALKING}

Controlling this model physically in real time may be difficult, because the control action is in 2D while the system is in fact in $3 \mathrm{D}$; that is, although this model might be stabilised in the frontal plane, lateral instability may arise. This discussion leads us to consider the design of advanced control to ensure fast tracking and robustness against endogenous unmodeled dynamics and external structured disturbances and, at the same time, a controller that is easy to implement, with an intuitive tuning procedure. Bearing this in mind, differential flatness (Fliess et al. 1995) and second-order sliding modes are synthesised (Vadim Utkin and Shi 1999; Parra-Vega 2001).

\section{Differential flatness}

According to Rouchon and Sira-Ramirez (2003), model (1)-(3) is flat; that is, there are two endogenous outputs that completely parameterise the system:

$$
\begin{aligned}
y_{1} & =\mathcal{F}_{0} \varphi_{0}+\mathcal{F}_{1} \varphi_{1}+\mathcal{F}_{2} \varphi_{2} \\
y_{2} & =\varphi_{2},
\end{aligned}
$$

with its associated differential parameterisation. If $m^{2}=$ $M g l$, equations (4) and (5) become

$$
\begin{aligned}
& y_{1}=\mathcal{F}_{0} \varphi_{0}+\mathcal{F}_{1} \varphi_{1}+\mathcal{F}_{2} \varphi_{2}, \quad \dot{y}_{1}=\mathcal{F}_{0} \dot{\varphi}_{0}+\mathcal{F}_{1} \dot{\varphi}_{1}+\mathcal{F}_{2} \dot{\varphi}_{2}, \\
& \ddot{y}_{1}=\mathcal{F}_{0} \ddot{\varphi}_{0}+\mathcal{F}_{1} \ddot{\varphi}_{1}+\mathcal{F}_{2} \ddot{\varphi}_{2} \triangleq M g l \sin \left(\varphi_{0}\right) \\
& y_{2}=w^{2} \sin \left(\varphi_{0}\right), \quad \dot{y}_{2}=\dot{\varphi}_{2}, \quad \ddot{y}_{2}=\ddot{\varphi}_{2} .
\end{aligned}
$$

This way, the states $\varphi_{0}, \varphi_{1}$, and $\varphi_{2}$ as a function of (4) and (5) produce

$$
\begin{aligned}
& \varphi_{0}=\arcsin \left(\frac{\ddot{y}_{1}}{w^{2}}\right), \quad \dot{\varphi}_{0}=\frac{y_{1}^{(3)}}{w^{2} \cos \left(\varphi_{0}\right)} \\
& \varphi_{1}=\frac{1}{\mathcal{F}_{1}}\left[y_{1}-\mathcal{F}_{0} \arcsin \left(\frac{\ddot{y}_{1}}{w^{2}}\right)-\mathcal{F}_{2} y_{2}\right], \\
& \dot{\varphi}_{1}=\frac{1}{\mathcal{F}_{1}}\left[\dot{y}_{1}-\mathcal{F}_{0} \frac{y_{1}^{(3)}}{\left.w^{2} \sqrt{1-\left(\frac{\ddot{y}_{1}}{w^{2}}\right)^{2}}-\mathcal{F}_{2} \dot{y}_{2}\right]}\right. \\
& \varphi_{2}=y_{2}, \quad \dot{\varphi}_{2}=\dot{y}_{2} .
\end{aligned}
$$

Therefore

$$
\ddot{y}_{1}-\mathcal{F}_{0}\left\{\frac{y_{1}^{(4)}}{w^{2} \sqrt{1-\left(\frac{\ddot{y}_{1}}{w^{2}}\right)^{2}}}+\frac{\left[\frac{y_{1}^{(3)}}{w^{2}}\right]^{2}\left(\frac{\ddot{y}_{1}}{w^{2}}\right)}{\left(1-\left(\frac{\ddot{y}_{1}}{w^{2}}\right)^{2}\right)^{\frac{3}{2}}}\right\}=\tau_{1}
$$

$\mathcal{F}_{2} \ddot{y}_{2}=\tau_{2}$.
That is, the system exhibits flatness with respect to the outputs $y=\left(\mathrm{y}_{1}, \mathrm{y}_{2}\right)$. The controls $\tau=\left(\tau_{1}, \tau_{2}\right)$ are now designed such that a canonical form of Brunovsky appears; that is, a flat inverse dynamics control $\tau$ is proposed as follows:

$$
\begin{aligned}
& \tau_{1}=\ddot{y}_{1}-\mathcal{F}_{0}\left\{\frac{v_{1}}{w^{2} \sqrt{1-\left(\frac{\ddot{y}_{1}}{w^{2}}\right)^{2}}}+\frac{\left[\frac{y_{1}^{(3)}}{w^{2}}\right]^{2}\left(\frac{\ddot{y}_{1}}{w^{2}}\right)}{\left(1-\left(\frac{\ddot{y}_{1}}{w^{2}}\right)^{2}\right)^{\frac{3}{2}}}\right\} \\
& \tau_{2}=\mathcal{F}_{2} v_{2},
\end{aligned}
$$

where $v_{1}$ and $v_{2}$ are auxiliary control inputs, designed such that the tracking is fast and robust.

\section{Second order sliding mode control}

For effective continuous biped walking, it is necessary to achieve fast convergence, even in the presence of endogenous disturbances not compensated by the flatness controllers $\tau_{1}$ and $\tau_{2}$. This can be achieved if we are able to introduce sliding modes without chattering through the auxiliary inputs $v_{1}$ and $v_{2}$, as follows:

$$
\begin{aligned}
v_{1}= & \left\{\left(y_{1}^{*}\right)^{(4)}-k_{4}\left(y_{1}^{(3)}-\left(y_{1}^{*}\right)^{(3)}\right)-k_{3}\left(y_{1}^{(2)}-\ddot{y}_{1}^{*}\right)\right. \\
& -k_{2}\left(\dot{y}_{1}-\dot{y}_{1}^{*}\right)-k_{1}\left(y_{1}-y_{1}^{*}\right) \\
& \left.-k_{i_{1}} \tanh \left(\lambda_{1} S_{1}\right)\right\}-k_{d_{1}} S_{e_{1}} \\
v_{2}= & \left\{\ddot{y}_{2}^{*}-k_{2}\left(\dot{y}_{2}(t)-\dot{y}_{2}^{*}\right)-k_{1}\left(y_{2}-y_{2}^{*}\right)\right. \\
& \left.-k_{i_{2}} \tanh \left(\lambda_{2} S_{2}\right)\right\}-k_{d_{2}} S_{e_{2}}
\end{aligned}
$$

The sliding mode surfaces $S_{1}=y_{1}^{(3)}-v_{1}$ and $S_{2}=$ $\dot{y}_{2}-v_{2}$ are

$$
\begin{aligned}
S_{1}= & \left(y_{1}^{(3)}-\left(y_{1}^{*}\right)^{(3)}\right)-k_{4}\left(y_{1}^{(2)}-\ddot{y}_{1}^{*}\right)-k_{3}\left(t y_{1}-\dot{y}_{1}^{*}\right) \\
- & k_{2}\left(y_{1}-y_{1}^{*}\right)-k_{1} \int_{0}^{t}\left(y_{1}(\sigma)-y_{1}^{*}(\sigma)\right) d \sigma \\
S_{2}= & \left(\dot{y}_{2}-\dot{y}_{2}^{*}\right)-k_{2}\left(y_{2}-y_{2}^{*}\right) \\
& -k_{1} \int_{0}^{t}\left(y_{2}(\sigma)-y_{2}^{*}(\sigma)\right) d \sigma
\end{aligned}
$$

such that the following extended surfaces arise

$$
\begin{aligned}
& S_{e_{1}}=S_{1}+k_{i_{1}} \int_{0}^{t} \operatorname{sgn}\left(S_{1}\right) d t \\
& S_{e_{2}}=S_{2}+k_{i_{2}} \int_{0}^{t} \operatorname{sgn}\left(S_{2}\right) d t
\end{aligned}
$$

for $\lambda_{1}>0, \lambda_{2}>0$ and positive definite feedback tuning $\left\{k_{4}, k_{3}, k_{2}, k_{1}, k_{d_{1}}, k_{d_{2}}, k_{i_{1}}, k_{i_{2}}\right\}$ of appropriate dimensions. Feedback gains have to be tuned such that all poles of this polynomial are placed in the left side of the complex halfplane. Substituting equations (10) and (11) and equations (12) and (13) into (8) and (9) yields the following closedloop error equation:

$$
\dot{S}_{e_{1}}=-k_{d_{1}} S_{e_{1}}+Z_{1}
$$




$$
\dot{S}_{e_{2}}=-k_{d_{2}} S_{e_{2}}+Z_{2},
$$

where $Z_{i}=\operatorname{sgn}\left(S_{e_{i}}\right)-\tanh \left(\lambda_{i} S_{i}\right)$ is bounded, for $i=1,2$. Since equations (18) and (19) yield an exponential envelope for $S_{e_{1}}$ and $S_{e_{2}}$, the derivative of equations (16) and (17), according to Parra-Vega (2001), guarantee exponential convergence of tracking errors $e(t)=y-y^{*}(t)$ owing to the existence of second-order sliding modes at $S_{1}=0$ and $S_{2}=0 \forall t$ as long as $k_{i}>\dot{S}_{e_{i}}$ for $i=1,2$.

Remark 1. Robustness. As shown in Parra-Vega (2001), when there are disturbances, the upper bound of $\dot{S}_{e_{i}}$ is higher. In this case, the proposed controller can still compensate disturbances simply by tuning $k_{i}>\dot{S}_{e_{i}}$ for $i=1,2$ (Vega 2001). In this case, in the presence of such disturbances, equations (20) and (21) become

$$
\begin{gathered}
\dot{S}_{e_{1}}=-k_{d_{1}} S_{e_{1}}+\zeta_{1} \\
\dot{S}_{e_{2}}=-k_{d_{2}} S_{e_{2}}+\zeta_{2},
\end{gathered}
$$

where $\zeta_{1}$ and $\zeta_{2}$ are bounded endogenous disturbances. Thus, if feedback gains $k_{i}>\dot{S}_{i}+\left\|\zeta_{i}\right\|(i=1,2)$, secondorder sliding modes are induced and exponential stability arises (Vega 2001).

Remark 2. Bandwidth. In practice, it is very likely that $\zeta$ appears due to the limited bandwidth of the system, quantisation of IO channels, PC processing, digital implementation of a continuous domain model, and electronic noise, as well as limited bandwidth of motor and actuators. Therefore, $k_{i}>\dot{S}_{e i}+\left\|\zeta_{i}\right\|(i=1,2)$ is preferred over $k_{i}>\dot{S}_{e i}(i=1,2)$.

\section{Path planning for continuous biped walking}

One of the advantages of implementing a differential flat controller is its duality to work as a path planner (Fliess et al. 1995, 1999). Here, the problem of path planning of walking patterns $\frac{d}{d t} y_{1}^{j, *}(j=1, \ldots, 4)$ is mapped to the subspace of flat outputs, satisfying all intermediate smooth end points and boundary constraints. In this way, we plan a stable periodic walking path from $\left[t_{0}, T\right]$, where $T$ is the time given for each step. For $t_{0}$, legs are opened symmetrically and the robot is in static equilibrium, such that at time $T$ the biped achieves one step; therefore we do have the relationships shown in Table 2. In this way, the following polynomial, for $\Delta(t)=\frac{t-t_{0}}{T-t_{0}}$, satisfies the constraints

$$
\begin{aligned}
y_{1}^{*}(t)= & \gamma_{1}+\gamma_{2} \Delta^{(1)}(t)+\gamma_{3} \Delta^{(2)}(t)+\gamma_{4} \Delta^{(3)}(t)+\gamma_{5} \Delta^{(4)}(t) \\
& +\gamma_{6} \Delta^{(5)}(t)+\gamma_{7} \Delta^{(6)}(t)+\gamma_{8} \Delta^{(7)}(t),
\end{aligned}
$$

such that boundary conditions are fulfilled, and finally, the linear system of equations are solved for $\gamma$. Now, it is necessary to plan the trajectory for $y_{2}$ such that $\left|\varphi_{0}\right| \leq$ $\left|\varphi_{2}\right| \forall t$; that is, the swing leg does not travel beyond the floor level. Therefore, we have

$$
y_{2}^{*}(t)=-f(t) \varphi_{0}^{*}(t),
$$

where $f(t)=1+\lambda(t)$, with $\lambda(t) \neq 0$ during the time interval $\left[t_{0}, T\right]$. Finally, to maintain continuous walking, leg
Table 2 Relationships between flat outputs

\begin{tabular}{ll}
\hline States at $t=t_{0}$ & States at $t=T$ \\
\hline$\varphi_{0}\left(t_{0}\right)=-\alpha$ & $\varphi_{0}(T)=\beta$ \\
$\varphi_{1}\left(t_{0}\right)=0$ & $\varphi_{1}(T)=0$ \\
$\varphi_{2}\left(t_{0}\right)=\alpha$ & $\varphi_{2}(T)=-\beta$ \\
$y_{1}\left(t_{0}\right)=\alpha\left(\mathcal{F}_{2}-\mathcal{F}_{0}\right)$ & $y_{1}(T)=\beta\left(\mathcal{F}_{0}-\mathcal{F}_{2}\right)$ \\
$\dot{y}_{1}\left(t_{0}\right)=y_{1}^{(3)}\left(t_{0}\right)=0$ & $\dot{y}_{1}(T)=y_{1}^{(3)}(T)=0$ \\
$\ddot{y}_{1}\left(t_{0}\right)=-w^{2} \sin (\alpha)$ & $\ddot{y}_{1}(T)=-w^{2} \sin (\beta)$ \\
$y_{2}\left(t_{0}\right)=\alpha$ & $y_{2}(T)=-\beta$ \\
$\dot{y}_{2}\left(t_{0}\right)=\ddot{y}_{2}\left(t_{0}\right)=0$ & $\dot{y}_{2}(T)=\ddot{y}_{2}(T)=0$ \\
\hline
\end{tabular}

Table 3 Biped robot parameters and feedback gains for simulations

\begin{tabular}{lll}
\hline Symbol & Value & Units \\
\hline$M$ & 0.40 & $\mathrm{~kg}$ \\
$l$ & 0.20 & $\mathrm{~m}$ \\
$\mathcal{f}_{0}$ & 0.02 & $\mathrm{kgm}^{2}$ \\
$\mathcal{F}_{1}$ & 0.0007 & $\mathrm{kgm}^{2}$ \\
$\mathcal{f}_{2}$ & 0.0005 & $\mathrm{kgm}^{2}$ \\
$T$ & 2 & $\mathrm{~s}$ \\
$T_{s}$ & 6 & $\mathrm{~s}$ \\
$\alpha$ & 0.391 & $\mathrm{rad}$ \\
$\beta$ & 0.391 & $\mathrm{rad}$ \\
$g$ & 9.81 & $\mathrm{~m} / \mathrm{s}^{2}$ \\
\hline
\end{tabular}

dynamics commutes each other, stance leg becomes swing leg and vice versa, and reset is carried out for the initial conditions of the interval $\left[T, T_{1}\right]$. Also, the path planning for $\varphi_{0}^{*}$ and $\varphi_{2}^{*}$ commutes each other. This discontinuity must be supervised carefully to avoid numerical inconsistencies.

\section{Numerical simulations}

A numerical simulator under Simulink-MATLAB Release 6 environment is implemented under a sampling period of $h=1 \mathrm{~ms}$, and without digital quantisation or limit switches. Parameters of the biped robot and feedback gains are given in Table 3 . Feedback gains $k_{i_{1}}=0.1, k_{i_{2}}=$ $0.15, k_{d_{1}}=50, k_{d_{2}}=6, \lambda_{1}=5, \lambda_{2}=5$.

Figure 3(a) shows how stance leg $\left(\phi_{a}\right)$ and swing leg $\left(\phi_{b}\right)$ tracks the desired trajectory for a three-step task. Each step lasts $2 \mathrm{~s}$; thus commutation of leg dynamics and controllers are carried out every $2 \mathrm{~s}$, when commutation occurs and the next step starts. Also, note that for each commutation of the legs, $\phi_{a}=\varphi_{0}, \phi_{b}=\varphi_{2}$ and invariably $\phi_{1}=\varphi_{1}$. Tracking errors are small, such that continuous biped walking occurs (Figure 3(b)). The rotary body experiences very high velocities of up to approximately $300 \mathrm{rad} / \mathrm{s}$, or near $3,000 \mathrm{rpm}$, in half step (time $t=T / 2$ ), that is, $1 \mathrm{~s}$. Notice that for the next $2 \mathrm{~s}$, the target is $3,000 \mathrm{rpm}$ in the other direction. This is caused by the very high torque difference $\tau_{a} \gg \tau_{b}$ (which is the input of the rotary body; see equation (2)) because $\tau_{a}$ (swing leg torque) pushes forward the whole biped robot with a fixed support point at the stance leg; thus $\tau_{\mathrm{b}}$ (stance leg torque) is very small since it only needs to balance the swing leg without any 

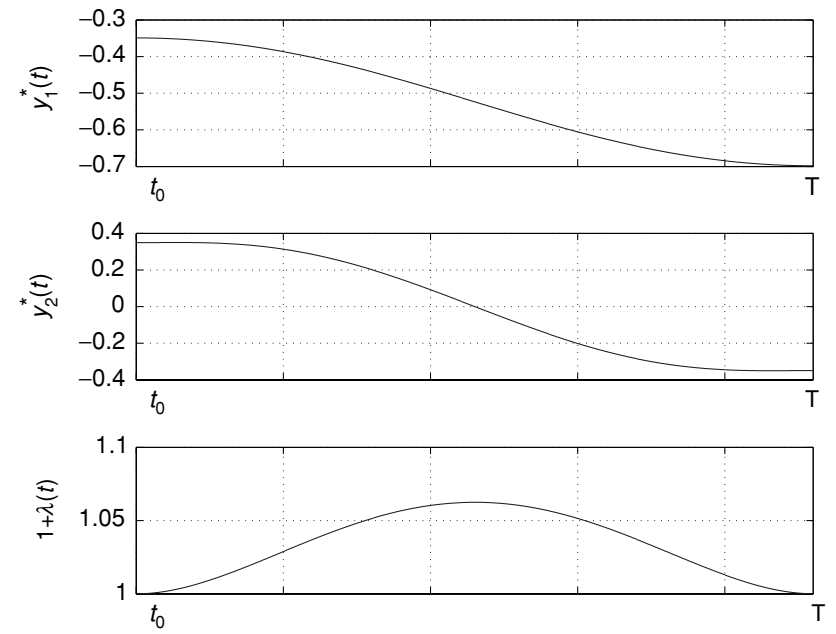

Figure 2 Path planning for $y_{1}^{*}(t)$ (first row) and $y_{2}^{*}(t)$ (second row); Function $f(t)=1+\lambda(\mathrm{t})$ (third row).

disturbance. In addition, note that the theoretical absence of friction in the model (there is no energy dissipation) produces such high velocities.

\section{Technological challenges}

To design the physical prototype, first of all, we need to discuss the $2 D$ simulation results, wherein technological challenges can be highlighted.

Remark 3. Rotary body velocities. The rotary body must achieve $3,000 \mathrm{rpm}$ in $1 \mathrm{~s}$, then go to near $0 \mathrm{rpm}$ in another second, and then change direction and achieve $3,000 \mathrm{rpm}$ in the next second, and so on. This regime is for slow walking, under the very strict assumption that the torque difference is transmitted without loss, and without impact at commuting, and with contact at $t=n T / 2$, for $n=1,2 \ldots$, the number of steps. This would require a new kind of actuator technology. Remember that this actuator is housed (or mounted) inside the rotary body, and so the reaction torque must be zero, which is unavailable yet in commercial off-the-shelf products.

Remark 4. Zero landing velocity. The biped robot dynamics says implicitly that the controller must guarantee zero landing velocity of the swing leg at the time of commuting, as that there is no impact. This would require a controller that, in practice and in theory, guarantees finitetime convergence at time $t=T / 2$.

Remark 5. Zero dynamic force coupling. Zero dynamic force coupling is not considered (Coriolis forces are neglected in the model), which in practice means that swing and stance leg dynamics are not really mechanically coupled, and that motion of one leg does not affect the motion of the other leg. This is not possible in practice, since this would require at least that the base of each inverted pendulum be independent. However, the base of both legs are attached to an inertial frame located in the rotary body. Thus, the assumption is not realistic.

All these remarks lead us to consider an advanced CAE tool to simulate a $2 \mathrm{D}$ walking behavior in a $3 \mathrm{D}$ world,
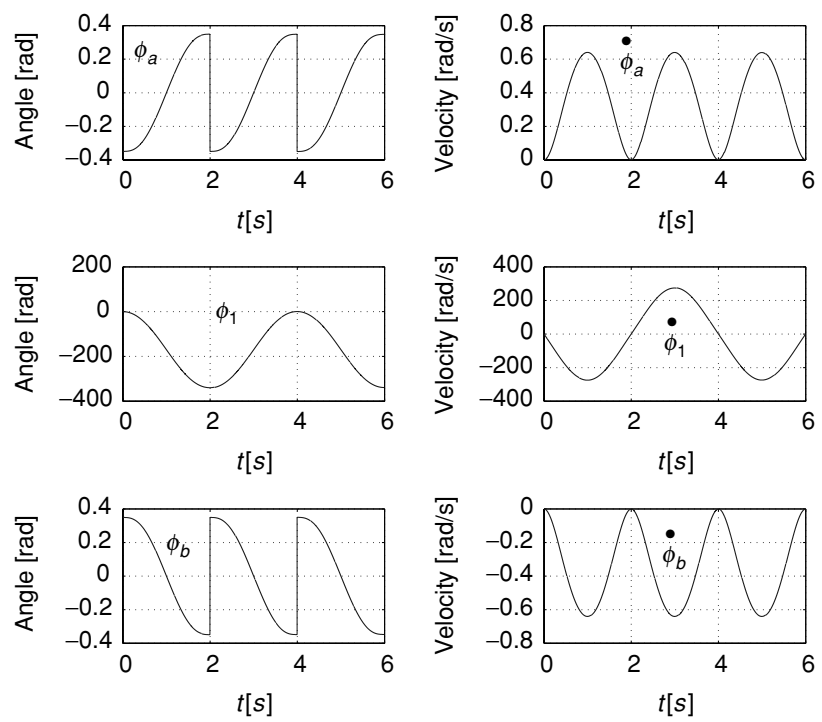

(a)
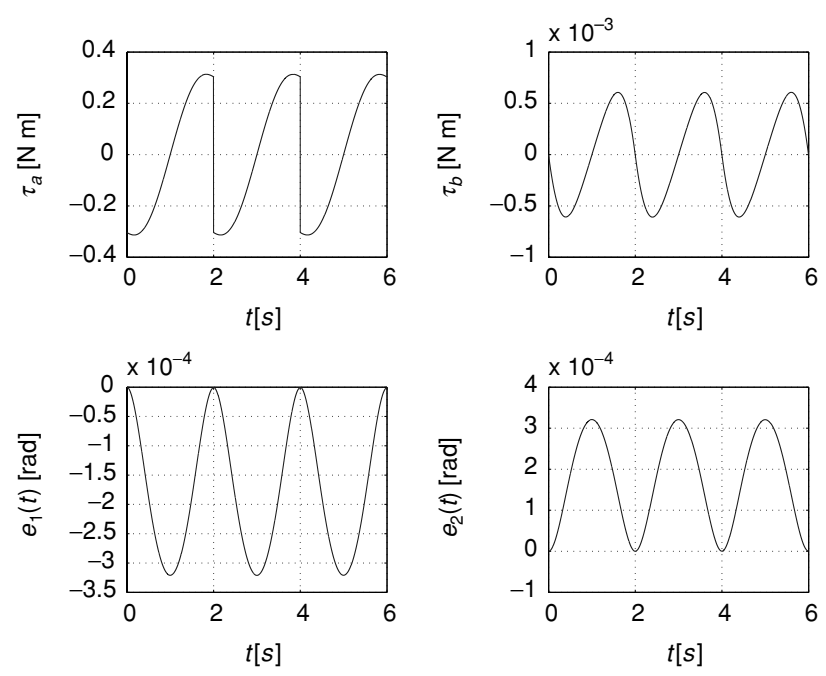

(b)

Figure 3 Simulations results for $T=6 \mathrm{~s}$; three steps are carried out. (a) States of stance leg, $\phi_{a}$, and swing leg, $\phi_{b}$, and rotary body, $\phi_{1}$. Notice that $\phi_{a}$ and $\phi_{b}$ commute each other at each step. (b) Torques (top row) and tracking errors (bottom row).

that is, by using solid components. To this end, there are several powerful commercial packages in the market that import 3D CAD, assign dynamic parameters, and then simulate under holonomic and nonholonomic constraints (e.g. SABER, ADAMS and CATIA). However, implementing this simulation in these packages requires a high level of dedicated knowledge; besides, these packages are very expensive and prohibitory for typical academic budgets. For this reason, and because we are interested in an open-architecture CAE simulator, we program and configure the modules required to interconnect several numerical packages to be able to integrate a mechatronics computer platform. The numerical packages such as Matlab, 


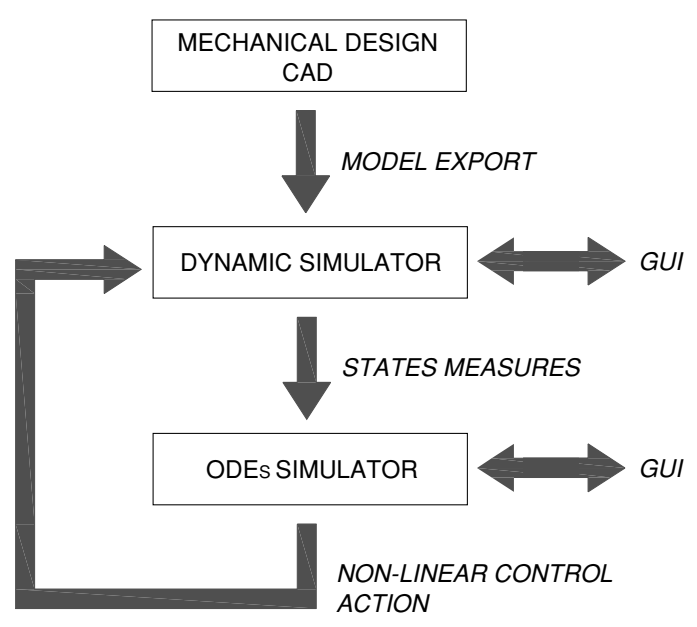

Figure 4 CAE simulator flow chart.

VisualNastran and Mechanical Desktop are easy to program by bachelor engineers.

\section{MECHATRONICS COMPUTER PLATFORM}

We are interested in a CAE simulator that is able to run in multiple domains (continuous and discrete) under a number of constraints (holonomic and nonholonomic) and under several time scales, and to resolve contact under many types of kinematic constraints. The basic characteristic of this simulator is that this tool must provide simulations of the CAD design. This will produce simulation results closer to reality in comparison to the numeric simulations of the previous section, and moreover will provide the preliminary design criteria of the kinematics, dynamics, the actuator and sensor specifications, as well the bandwidth of the computer platform. Thus, we would like to define the computational mechatronics concept as 'Modeling and Simulation of Dynamic Systems, Based on Applied Mechatronics Scheme on a CAE Computational Platform', that is, the concurrent virtual integration of mechanical systems with electrical-electronic systems having non-linear and linear applied control via a computer platform. The new scheme includes the following:

- Mathematical and geometrical modeling (CAD) using hardware integration of the dynamic system under examination.

- Analysis and synthesis of applied control techniques to mechanical design, commonly known as CAD/CAM.

- Emulation of software integration, data acquisition, electronic constraints, and finally constraints of sensors and actuators.

Three software packages were integrated to form a unique computational platform that lets us introduce a computational mechatronic approach, called the CAE simulator: Mechanical Desktop for the CAD design, VisualNastran 4D for the assignment of constraints as well as the numerical solvers and MATLAB-Simulink release 6.0 as the controller platform (see Figure 4). This CAE simu-

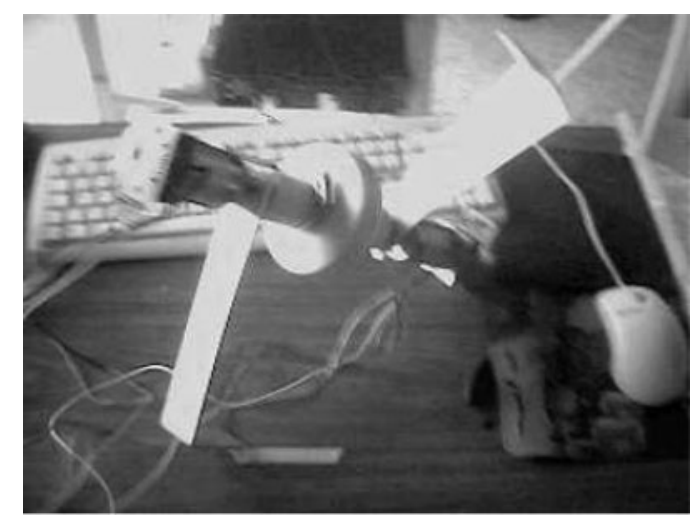

(a)

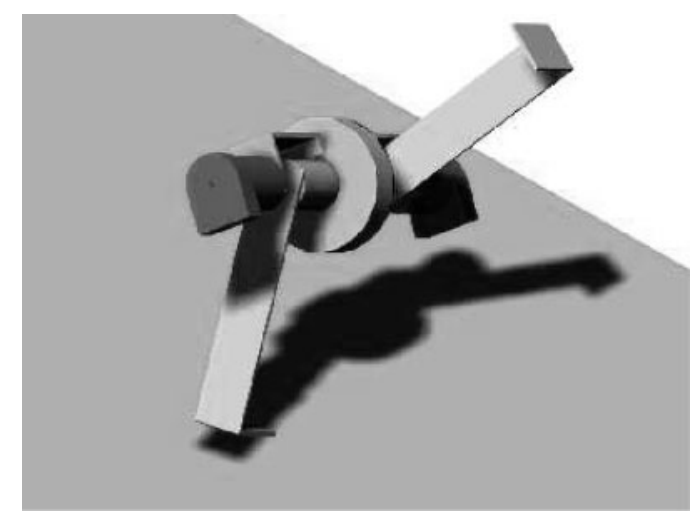

(b)

Figure 5 Real-time and CAE simulator results: Notice the very well-matched results. The CAE simulator uses the real parameters obtained through the use of real constraints and the quantisation of the real system. (a) Instability due to torsional torques along the $\vec{z}$ axis. (b) The CAE simulator reproduces the above fall, thanks to implementation of the mechatronics approach.

lator provides the opportunity to introduce realistic constraints, material properties, working conditions, quantisation input-output data, quantisation, limited bandwidth, signal conditioning and multiscale sampling rates, as well as mechanical failure. Rather than a simulator, it is a powerful computer emulator system for a qualified engineer to have at hand. Figure 5 shows snapshots with a not surprising match between real behavior and computer CAE simulations, under harsh and extreme conditions. It is not surprising because a mechatronics approach has been considered.

\section{THE REAL BIPED ROBOT PROTOTYPE}

Simulation results of the previous section provided the parameters for designing a real biped robot using a mechatronics approach: 


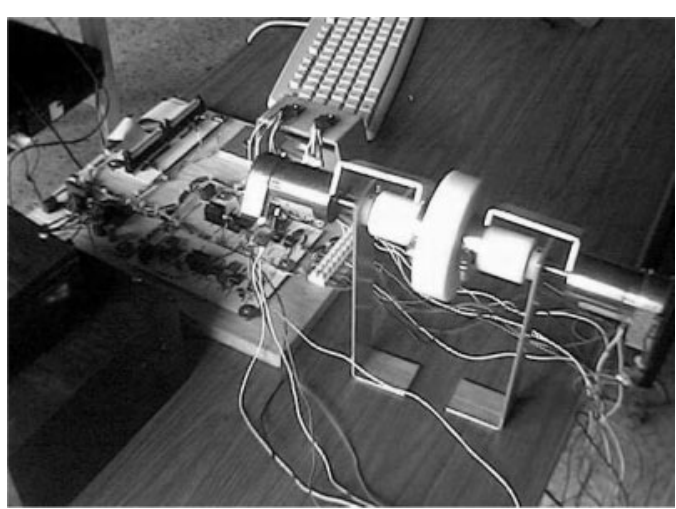

Figure 6 Preliminary experimental prototype.

Table 4 Biped (real) robot parameters (left) and feedback gains (right)

\begin{tabular}{lll}
\hline Symbol & Value & Units \\
\hline$M$ & 0.4523 & $\mathrm{~kg}$ \\
$l$ & 0.20 & $\mathrm{~m}$ \\
$\mathcal{F}_{0}$ & 0.0187 & $\mathrm{kgm}^{2}$ \\
$\mathcal{F}_{1}$ & 0.0008 & $\mathrm{kgm}^{2}$ \\
$\mathcal{F}_{2}$ & 0.0006 & $\mathrm{kgm}^{2}$ \\
$T_{s}$ & 2 & $\mathrm{~s}$ \\
$T_{e}$ & 10 & $\mathrm{~s}$ \\
$\alpha$ & 0.391 & $\mathrm{rad}$ \\
$\beta$ & 0.391 & $\mathrm{rad}$ \\
$g$ & 9.81 & $\mathrm{~m} / \mathrm{s}^{2}$ \\
\hline
\end{tabular}

- CAD design

- Sensor and actuator considerations

- Electronic and microelectronic circuits

- Hardware integration

- Software integration

- Integration

The mechatronics approach provides a unique platform to integrate the hardware and software characteristics, capacities and constraints at every instant-in contrast to the traditional approach, wherein every subsystem is designed using inputs and outputs from the other interconnected subsystems.

This design suggests using permanent-magnet 12-V DC motors of $0.5 \mathrm{Nm}$ torque, encoders of $512 \mathrm{CPR}$, clean and robust measurement of velocity and green nylamid for the rotary body, with aluminium legs. The motors are fixed to the base attached to the free-floating rotary body. The parametric dimensions of the system were obtained such that the torque and velocity of the rotary body are minimised. The following problems were encountered:

(1) Friction-free floating body cannot be implemented.

(2) The torque input of the swing leg is very high because torque cannot be transmitted without loss.

(3) When the biped robot is in the air, reaction forces between both controllers (both control inputs are attached to the same frame) cannot comply to the model.
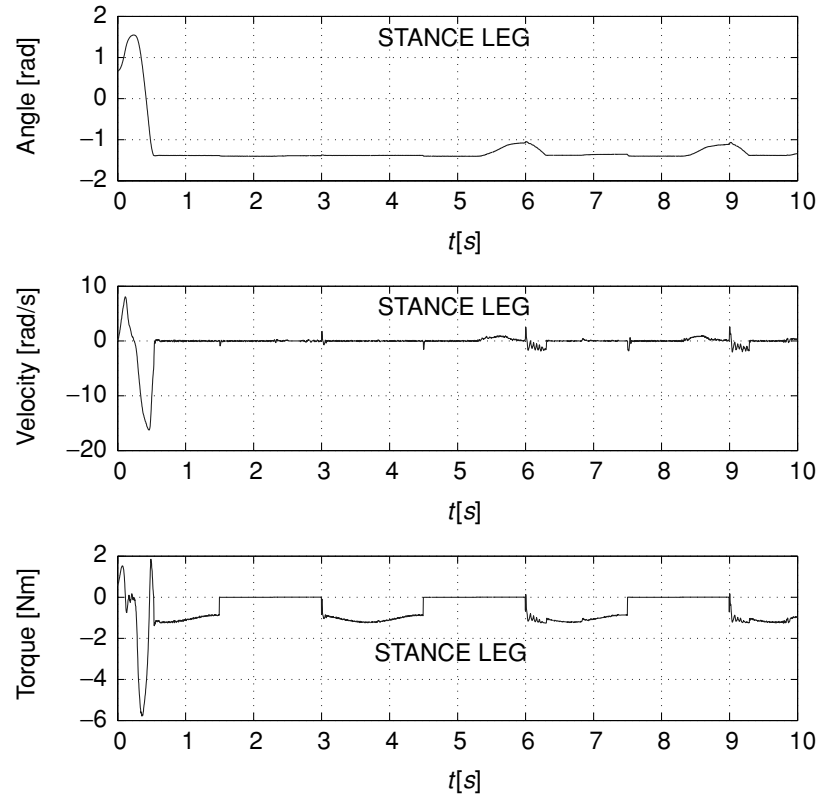

(a)
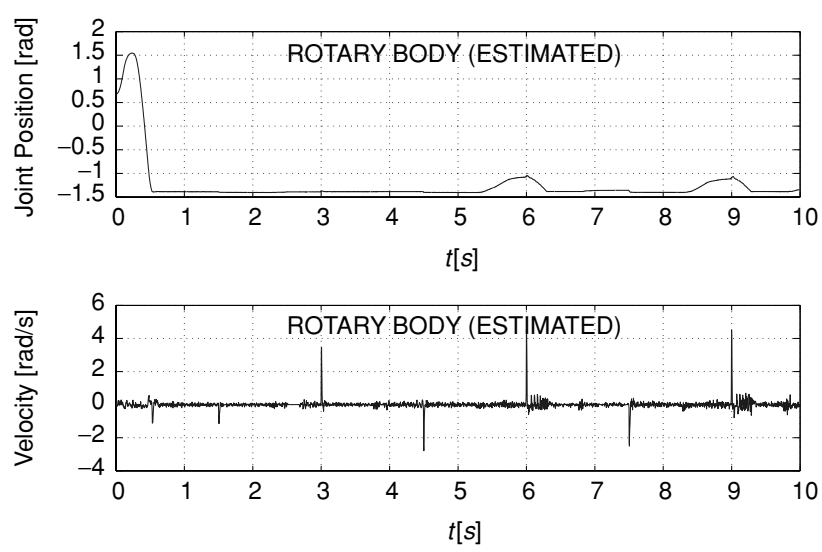

(b)

Figure 7 Experimental results for $T e=10$ s. (a) States response for stance leg. (b) States response for central body.

(4) An encoder needs to measure the joint position of the rotary body, $\varphi_{0}$. However, the rotary body is a freefloating element.

(5) The angle between the stance leg and the swing leg can be determined algebraically using the relation $\varphi_{0}=$ $\left|\varphi_{2}\right|-\left|\varphi_{1}\right|$. Although possible according to the model assumptions, in practice, this model is not real, and thus real $\varphi_{0}$ cannot be calculated in this way.

(6) This algebraic method is theoretically valid since it does not alter the tracking of flat outputs, because $\varphi_{0}$ is invariant with respect to $\varphi_{1}$ and $\varphi_{2}$. However, this not true in practice.

\section{EXPERIMENTAL RESULTS}

A standard Pentium III PC running at $466 \mathrm{MHz}$ under Windows 98 was used as the computer platform. An ISA IO card from Humusoft MF604 at 14 bits with 

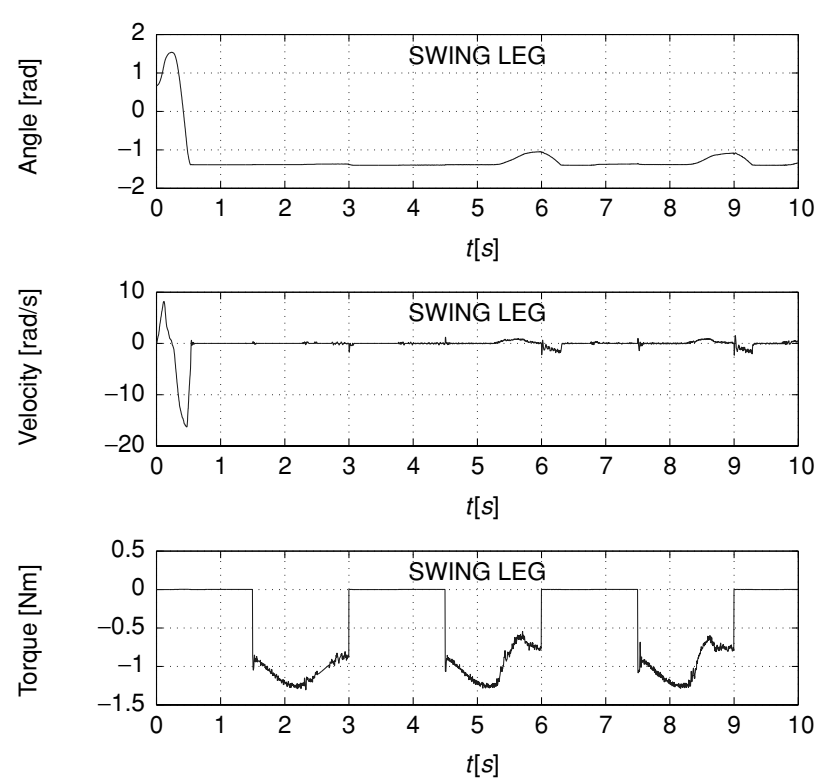

(a)
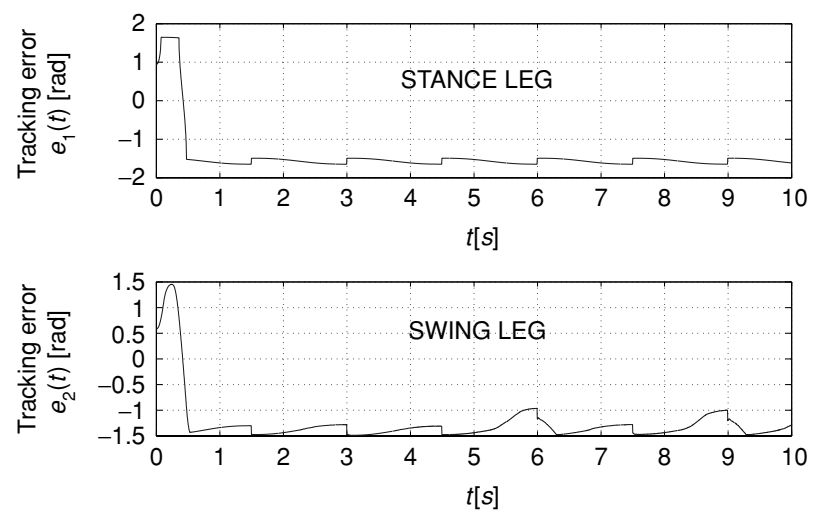

(b)

Figure 8 Experimental results for $T e=10$ s. (a) States response for swing leg. (b) Tracking errors response.

quadrature encoders of $512 \mathrm{CPR}$ read the joint position of the biped robot. Feedback gains were as follows: $k_{i_{1}}=0.1 ; k_{i_{2}}=0.15 ; k_{d_{1}}=50 ; k_{d_{2}}=6 ; \lambda_{1}=5 ; \lambda_{2}=5$. Table 4 shows the parameters of the biped robot shown in Figure 4(a). These parameters were estimated using a variety of methods in the laboratory. Figure 7 shows the position, velocity, torque and tracking error for each leg when the biped robot is on the floor.

Figures 5, 7 and 8 show that the biped robot falls owing to lateral instability, which arises because, basically, this is a $3 \mathrm{D}$ system and the controller is designed for a $2 \mathrm{D}$ system. Since Coriolis forces are not included in the model, and both inverted pendulums are attached to the same inertial frame, the torsional torques along $\vec{z}$ causes instability.

On the other hand, to really eliminate contact force and impact, the biped robot is suspended; that is, the biped robot rests on a parallel bar. Thus the biped robot in reality consists of two inverted pendulums linked by a high-velocity rotary body. In this case, the reaction forces destabilises the system because it was not modeled as two inverted pendulums. All these effects do appear in the CAE simulator.

\section{CONCLUSIONS}

A mechatronics-based design and analysis of a very simple biped robot for continuous active walking is presented. This study highlights several issues, ignored previously in biped models, that have arisen on using a computational mechatronics scheme. Experimental results suggest that the non-linear couplings and friction of a real planar biped walking system cannot be ignored; otherwise model reduction leads only to interesting academic exercises, but with very poor implications for practitioners, even when very advanced control design is introduced-in this case a differential flatness-based and chattering-free second-order sliding mode controller, which achieves fast and robust control.

\section{ACKNOWLEDGMENTS}

V. Parra-Vega acknowledges support from CONACYT project 47292-Y.

\section{REFERENCES}

Fantoni I, Lozano R. 2002. Non-linear Control for Underactuated Mechanical Systems. London: Springler-Verlag.

Fliess M, Levine J, Martin P, Rouchon P. 1995. Flatness and defect of nonliner systems: Introductory theory and examples. Int $\mathcal{F}$ Control, 61(6):1327-61.

Fliess M, Levine J, Martin P, Rouchon P. 1999. A Lie-Bäcklund approach to equivalence and flatness of nonlinear systems. IEEE Trans Automat Control, 44(5):992-37.

González-Hernández HG. 2004. Experimental analysis and control of a chaotic pendubot. Int 7 Robotics Res, 23(9):891-901.

Grizzle J. 2001. An analytical approach to asymptotically stable walking in planar biped robots. In Super Mechano Symposium Conference, November 19-21.

Grizzle J, Abba G, Plestan F. 2000. Asymptotic stability of a walking cycle for a biped robot with knees and torso. In NATOAVT Fall Symposium, Ankara Turkey.

Grizzle J, Abba G, Plestan F. 2001. Asymptotic stable walking for biped robots: Analysis via systems impulse effects. IEEE Trans Automat Control 46:51-64.

Grizzle J, Plestan F, Abba G. 2002. Poincaré's method for systems with impulse effects application to mechanical biped locomotion. Proceedings of the 1999 IEEE-CDC Conference, Phoenix, AZ, December 1999.

Ioi K, Igarashi H, Murakami A. 2002. Design of a gravitational wheeled robot. Adv Robot VSP Robot Soc Jpn, 16:785-93.

Kieffer J, Bale R. 1993. Walking viability and gate synthesis for a novel class of dynamically simple bipeds. Informatica, 17:144-55. 
McGee TG, Spong M. 2001. Trajectory planning and control of a novel walking biped. In IEEE Conference on Decision and Control, México City, pp. 1099-104.

Plestan F, Grizzle JW, Westervelt E, Abba G. 2001. Controlled periodic motion in a nonlinear system with impulse effects: Walking of a biped robot. In IFAC NOLCOS, Saint-Petersburg, Russia.

Raibert M. 1986. Legged Robots That Balance. The MIT Press Series in Artificial Intelligence. Cambridge, MA: MIT Press.

Rouchon P, Sira-Ramirez HJ. 2003. Control of the walking toy: A flatness approach, IEEE Am Control Conf, 3:2018-23.

Spong MW, Lozano R, Mahony R. 2000. An almost linear biped. In proceedings of the IEEE Conference on Decision and Control, Sydney, Australia, December 2000, pp. 4803-08.

Vadim Utkin JG, Shi J. 1999. Sliding Mode Control in Electromechanical Systems. Philadelphia: Taylor \& Francis.

Vega VP. 2001. Second order sliding mode control for robot arms with time base generators for finite-time tracking. Dynamics and Control, 11(2):175-86.

Westervelt ER, Grizzle JW. 2002. Design of asymptotically stable walking for a 5 -link planar biped walker via optimization. In ICRA-2002, May 10-17, Washington, DC.

Zheng YF, Hemami H. 1984. Impact effects of biped contact with the environment. IEEE Trans Syst Man Cybernet 21(3):437-43. 

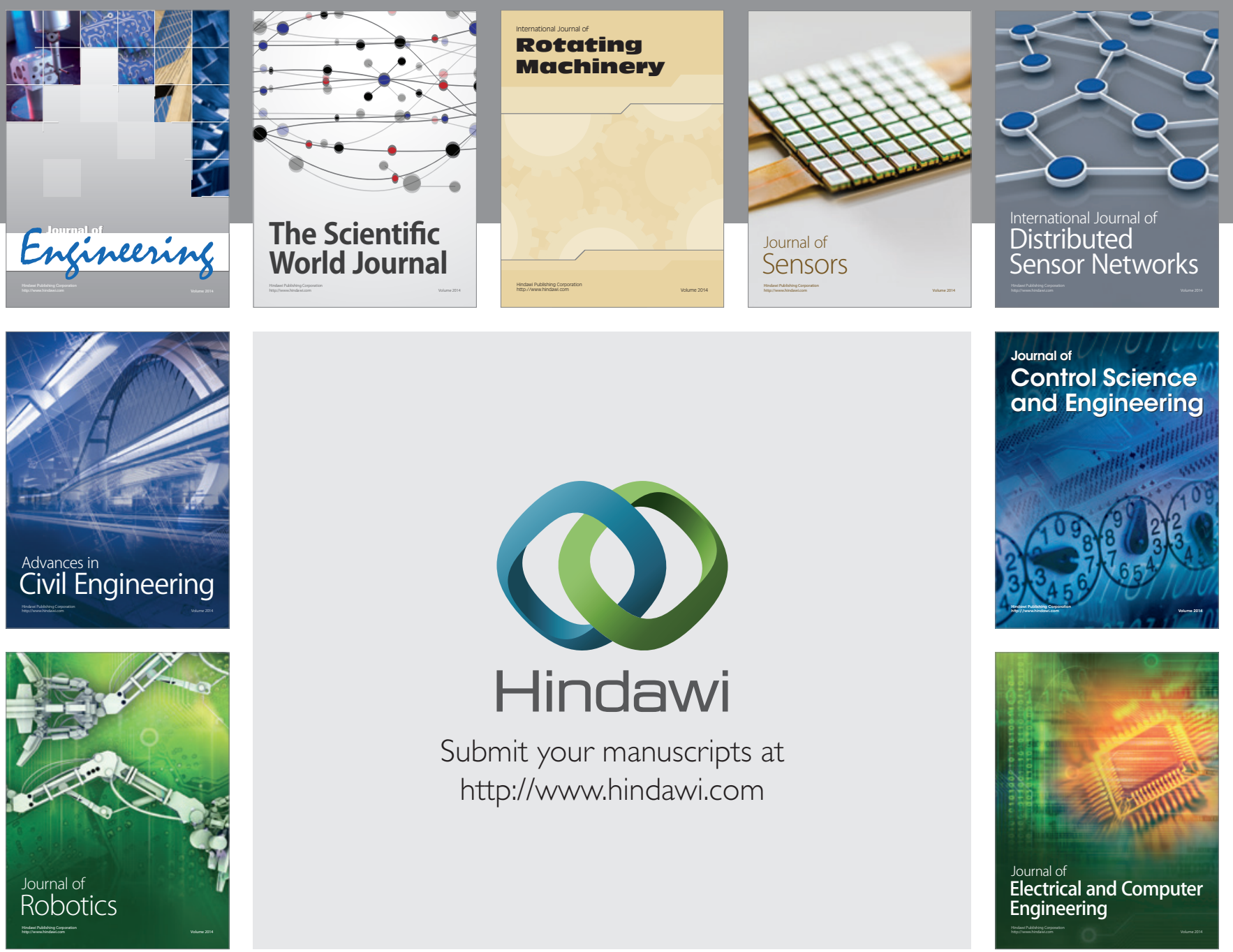

Submit your manuscripts at

http://www.hindawi.com
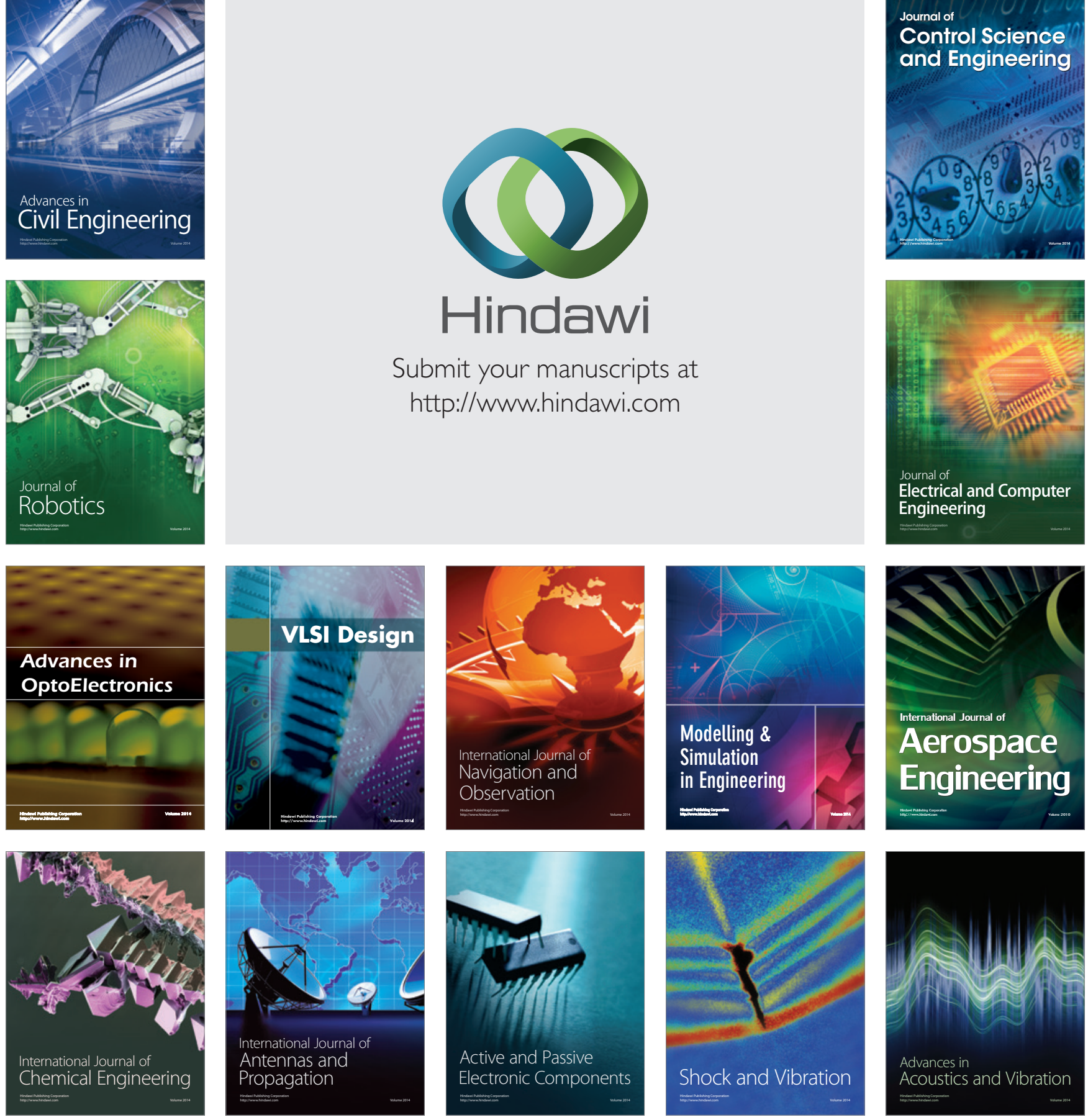